\title{
Development of a Novel System for Adaptive Machining of Near-Net-Shape Components
}

\author{
Zhengcai Zhao ${ }^{\mathrm{a}, \mathrm{b}, 1}$ Shengtao Lin ${ }^{\mathrm{a}}$, Yucan $\mathrm{Fu}^{\mathrm{a}, \mathrm{b}}$ \\ ${ }^{a}$ College of Mechanical and Electrical Engineering, Nanjing University of Aeronautics \\ and Astronautics, Nanjing 210016, China \\ b Jiangsu Key Laboratory of Precision and Micro-Manufacturing Technology, Nanjing \\ University of Aeronautics and Astronautics, Nanjing 210016, China
}

\begin{abstract}
Near-net-shape components are popular among the aerospace industry for low material waste and high manufacturing efficiency. However, it is difficult to machine such components into final shapes because the machining allowance is often distributed unevenly and even insufficient. This paper proposed a novel system for adaptive machining near-net-shape components, which integrates units like onmachine measurement based on probe and ultrasonic-sensor, machining allowance constrained localization, tolerance range constrained shape reconstruction, and TCP (tool cutter position) template-based NC programming. Firstly, localization and free form deformation (FFD)-based shape construction are performed within the tolerance ranges of the component, and an even distribution of the machining allowance can be obtained. Next, the quick NC programming that directly manipulates the TCPs by using spatial deformation is introduced. Last, the data transmission between units is illustrated. A case study of the machining titanium turbine blade is performed, which validates the proposed system.
\end{abstract}

Keywords. Adaptive machining system, shape reconstruction, near-net-shape.

\section{Introduction}

Near-net-shape components, such as aircraft skins and engine blades, are widely adopted in the aerospace field. However, their machining allowance is often unevenly distributed and even insufficient, which makes it likely to cause overcutting or undercutting by applying the NC program generated from design models. To address the above difficulties, geometrical adaptive machining (GAM) is usually adopted as it can flexibly adjust machining schemes according to the component features. GAM usually includes on-machine measurement, localization, shape reconstruction, and NC programming, etc.

The shape of the blank is an important basis for shape reconstruction and machining allowance analysis. On-machine measurement using the trigger probe [1] is an effective method to measure the shape of the blank, whose result is in the form of the point cloud. Before the near-net-shape component is machined, it is imperative to check its machinability. The localization [2] process is used to search the optimized point-pair

\footnotetext{
${ }^{1}$ Corresponding Author, Zhengcai Zhao, College of Mechanical and Electrical Engineering, Nanjing University of Aeronautics and Astronautics, Nanjing 210016, China; E-mail: zhengcaizhao@nuaa.edu.cn.
} 
relationship between the measured blank and the design model, which enables the blank to encompass the design model. For near-net-shape components, sometimes, deformations are beyond localization, which means under no circumstance can the measured blank completely encompass the design model, then the shape reconstruction of the design model is needed. The reconstruction [3] can be a local or global modification of the design shape, after which the modified design model can be encompassed by the measured blank and be used to generate tool paths.

To make the most of adaptive machining, these GAM units should be combined into an integrated system and their data needs to be processed efficiently. This paper investigates the methods and strategies of GAM and proposes a novel GAM system (shown in Fig 1) for machining the near-net-shape components in aerospace applications. The on-machine measurement of profile and thickness is not introduced in this paper. The rest of the paper is laid out as follows. In section 2, the overview of GAM is elaborated and the proposed GAM system is presented. In section 3, the machining allowance constrained localization is given, the FFD-based shape reconstruction method is illustrated, and quick NC programming using FFD is introduced. In section 4, the integration and the data transmission of the system are presented. An experiment of machining the titanium turbine blade is carried out to validate the proposed GAM system. Finally, in section 5, the conclusions of the proposed adaptive machining system are discussed.

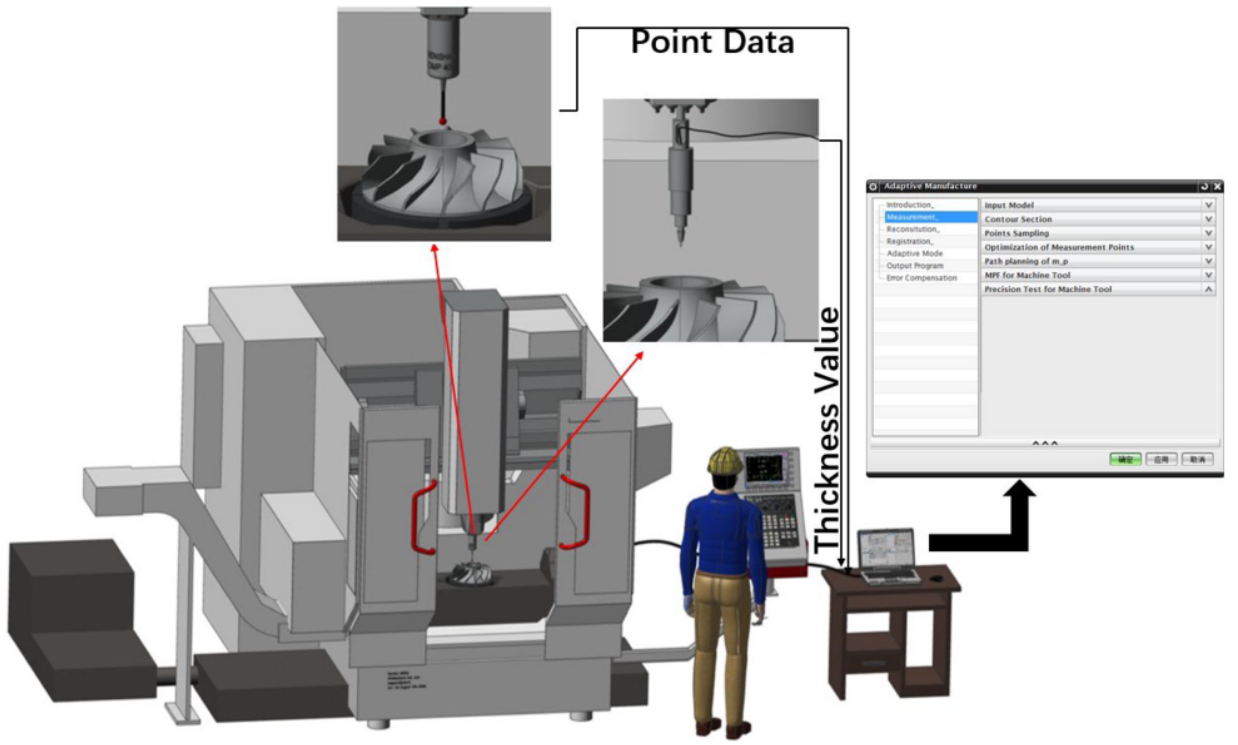

Figure 1. Overview of the proposed GAM system.

\section{Overview of GAM System}

GAM is a general description for a certain class of machining approaches, which are developed for meeting various specific requirements of the parts. The requirements may include part localization, machining allowance optimization, quick NC programming for the near-net-shape of the parts. 
The proposed GAM system consists of numerical machine tools, measuring sensors, a workstation with an analyzing software package, and some facilities for data transmission. A trigger probe and an ultrasonic sensor are integrated into the machine tools and can be driven by the NC system for inspecting the profile and thickness of the near-net-shape component. A high-performance workstation is employed to run the software package for analyzing the measured results. The algorithms of measurement planning, part localization, machining allowance optimization, quick NC programming are developed on the platform of this software package. Besides, the transmission of the measured data and NC programs is performed automatically within the GAM system by using OLE for Process Control (OPC) technology. The measured data involving the profile points and the thickness values are collected from the machine tool and the ultrasonic sensor and are transmitted to the workstation. The software package outputs the NC programs which are transmitted to the NC system of the machine tool to drive the sensors and tools for inspection and to perform the machining operation.

\section{Localization, Shape Construction, and Quick NC Programming}

\subsection{Localization}

To determine the proper mapping between the measured blank and the part CAD model, the modified classic iterative closest point (ICP) algorithm is adopted for preliminarily computing the rotational and translational matrices, which indicate the relative transformation relationship between measured data and part model.

Assume that $t_{1}$ and $t_{2}$ are the upper and lower band of the profile tolerance, and $d_{i}$ is the directed distance between the measured point and the design part profile. If $\mathrm{d}_{\mathrm{i}}<\mathrm{t}_{2}$, the part cannot be machined due to the lack of material on the blank, then a constrained ICP algorithm is proposed to further solve the problem of insufficient machining allowance, which can be formulated as:

$$
\left\{\begin{array}{l}
\text { Minimize } \sum_{i=0}^{N_{p}}\left\|P_{i}-R M_{i}-T\right\|^{2} \\
\text { s.t. } D_{i}=d_{i}+t_{2}>0
\end{array}\right.
$$

where $D_{\mathrm{i}}>0$ is the restriction to keep the measured blank encompassing the design part and thus ensure the machinability of the blank after the localization process. By solving Eq. (1), the optimized $\mathrm{R}$ and $\mathrm{T}$ are used to align the coordinate system of the part CAD model with that of the measured blank, and the machining allowance of the blank can be calculated.

\subsection{Shape Construction}

The tolerance band is set to allow the slight variation of the final part shape compared to the design shape. Based on this principle, the FFD-based shape construction is proposed to adaptively reconstruct a qualified shape of the design part. The free-form deformation (FFD) technology can construct a parametric volume which is defined by a 3D lattice of control points [4]. In the parametric volume, the design part model is embedded and then 
deformed. After the design part model is modified, the measured blank will be matched with the modified part model again to check its machinability.

The FFD we employ in this paper is a trivariate tensor product B-spline function, which is defined as

$$
d_{f f d}\left(s_{i}, t_{j}, u_{k}\right)=\sum_{i=0}^{L} \sum_{j=0}^{M} \sum_{k=0}^{N} g_{i, j, k} B_{i}\left(s_{i}\right) B_{j}\left(t_{j}\right) B_{k}\left(u_{k}\right)
$$

where $s_{i}, t_{i}, u_{k}$ are the local coordinate of the point embedded in the volume, L, M, N are the numbers of lattice grids, $g_{i, j, k}$ is the $\mathrm{i}^{\text {th }}, \mathrm{j}^{\text {th }}, \mathrm{k}^{\text {th }}$ control point in the $\mathrm{s}, \mathrm{t}, \mathrm{u}$ direction respectively. $B_{i}\left(s_{i}\right), B_{j}\left(t_{j}\right), B_{k}\left(u_{k}\right)$ are the B-spline blending functions.

To modify the design part model, the displacement $\varepsilon_{i, j, k}$ is imposed on the control point $g_{i, j, k}$ within the FFD volume, of which the deformation can be expressed as:

$$
d_{a}=M-P_{d}=\sum_{i=0}^{L} \sum_{j=0}^{M} \sum_{k=0}^{N} \varepsilon_{i, j, k} B_{i}\left(s_{i}\right) B_{j}\left(t_{j}\right) B_{k}\left(u_{k}\right)
$$

where $\mathrm{M}$ is the measured point, and $P_{d}$ is the modified point of the part model that ensures the desired machining allowance $d_{a}>0$ after the localization process. As $\varepsilon_{i, j, k}$ is solved, the deformed FFD volume is obtained, into which the qualified shape of the part is acquired by being embedded.

\subsection{Quick NC Programming}

Once the shape of the design part is reconstructed, the $\mathrm{NC}$ machining programs need to be regenerated. Therefore, a quick NC programming method based on FFD is proposed. The generated from the design part are adopted for the contribution to reducing repetitive programming, which is already post-processed.

The method of quick NC programming can be described as follows. First, the design blank and the measured blank are localized to calculate the FFD volume. Second, an iterative process is adopted to refine the FFD volume if its deformation error is greater than the given threshold. Then if not, the calculated FFD volume is imposed on the template TCPs to obtain the modified one. Third, a smooth Bezier surface is constructed between the modified TCPs and the measured blank, onto which the modified TCPs are projected for realizing a smooth transition of TCPs between certain areas of the blank. Finally, the projected TCPs are used to overwrite the corresponding information in the template TCPs file to obtain adaptive NC programs, which are automatically completed in the CAM software.

\section{System Integration and Case Study}

\subsection{System Integration}

The data transmission in the integrated GAM system can be described as follows. First, the on-machine measurement module transmits the measurement procedures to the 
machine system, then the measured data acquired from measuring sensors that are integrated on the machine tool would be transmitted back to on-machine measurement. Next, the measured data would also be transmitted to the reconstruction module, localization module, and error compensation module respectively. In the reconstruction module, the measured data can be used to establish a modified part model that can be applied to generate modified TCPs using constrained shape construction. In the localization module, the measured data can be aligned with the design part model to obtain the point-pair relationship and calculate the machining allowance of the blank. Then the point-pair relationship would be transmitted to the adaptive deformation module and automatic CNC programming module respectively, in which the FFD of the design part model can be operated, and the template TCPs can be modified and postprocessed as $\mathrm{CNC}$ machining programs to perform machining of blank on $\mathrm{CNC}$ machine tool. Finally, when the machining of the part is finished, the measurement would be performed again and the measured data would be transmitted to the error compensation module, in which the profile accuracy and thickness accuracy of the part would be calculated to determine whether the part needs to go through the process of adaptive machining again.

\subsection{Case Study}

To validate the feasibility of the proposed novel GAM system, an example of a certain hollow titanium alloy blade milling is given. The GAM system and the blank of the blade are presented in Fig 2.
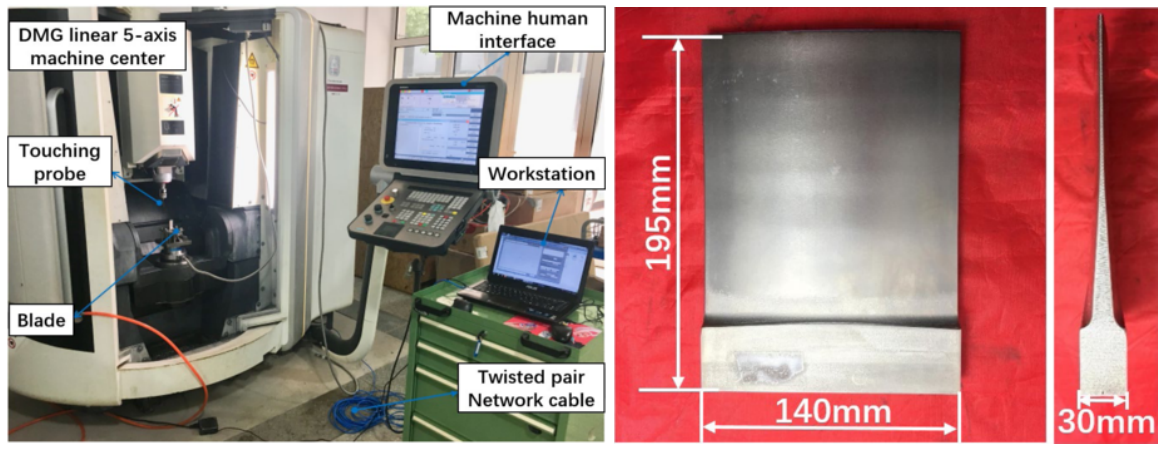

Figure 2. GAM system and the blank of the blade.

After the measured (leading and trailing edges) LTE data of the blade and design part model are put through the localization module, the error between them ranges from -0.969 to $0.833 \mathrm{~mm}$ (see Fig 3(a)), which indicates that the LTE of blank lacks machining allowance. Then, the part model has been modified using the proposed shape construction method, and the error result ranges from -0.000 to $1.413 \mathrm{~mm}$ (see Fig 3(b)), which means the machining allowance is enough and the blank of the blade is machinable.

The LTE of the blade is machined only once and has already met the requirements. The experiment result (see Fig 4) shows that the transition area from LTE to the blade body is smooth. The profile error of machined LTE is measured on Zeiss Prismo CMM, which ranges from $-0.042 \sim 0.072 \mathrm{~mm}$. The average roughness is measured as Ra $0.9 \mu \mathrm{m}$ 
using Mahr PS1 roughness tester. The proposed GAM system and the algorithms are tested to be feasible.

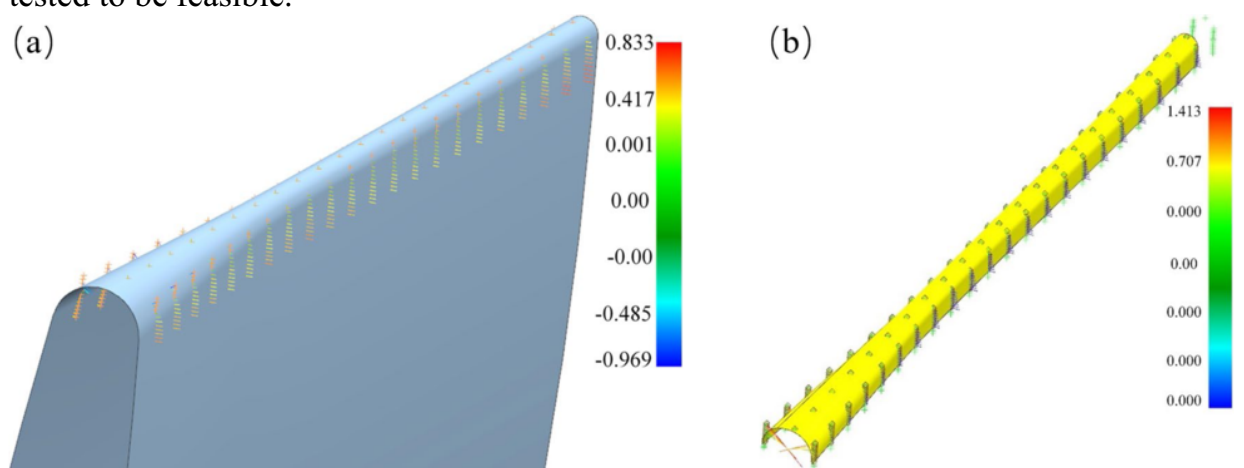

Figure 3. Localization result between the measured blank and part model.

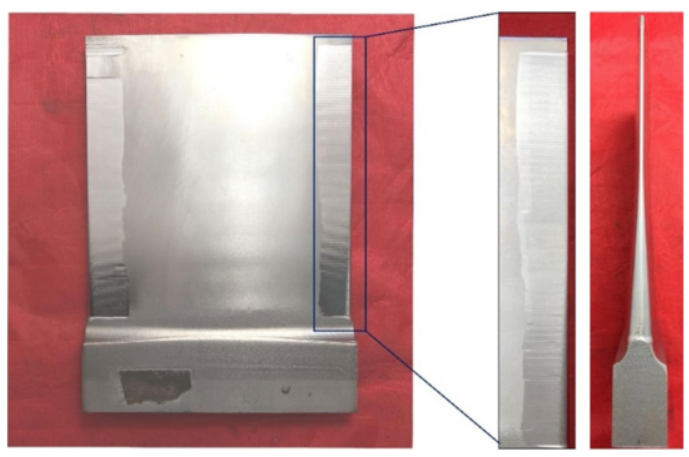

Figure 4. Machining result of the LTE by using the proposed GAM system.

\section{Conclusions}

In this paper, a novel geometrical adaptive machining system is developed for machining near-net-shape components in aerospace applications, which especially can solve the problems of difficult positioning and non-uniform machining allowance distribution. The developed GAM system is verified by the LTE cutting experiment. The profile error ranges from $-0.042 \sim 0.072 \mathrm{~mm}$, and the average roughness value is $0.9 \mu \mathrm{m}$, which both meet the machining requirements of LTE.

\section{References}

[1] Gaiyun H, Yicun S, Hongliang W. A profile error evaluation method for freeform surface measured by sweep scanning on CMM. Precision Engineering. 2019 Mar;56:280-292.

[2] Ying Z, Dinghua Z, Baohai W. An approach for machining allowance optimization of complex parts with integrated structure. Journal of Computational Design and Engineering. 2015 Otc;2:248-252.

[3] Benoit C, Sylvain P. An efficient EM-ICP algorithm for non-linear registration of large 3D point sets. Computer Vision and Image Understanding. 2020 Feb;191:102854.

[4] Yazhou F, Junxue R, Yongshou L. Prediction and reconstruction of edge shape in adaptive machining of precision forged blade. International Journal of Advanced Manufacturing Technology. 2018 Feb;96:2355-2366. 